\title{
An international interdisciplinary graduate school in laser and material science
}

Evelyne Fargin, Laurent Sarger, Malte Kaluza, Stefan Nolte, Martin Richardson, et al.

Evelyne Fargin, Laurent Sarger, Malte Kaluza, Stefan Nolte, Martin

Richardson, Kathleen Richardson, "An international interdisciplinary graduate school in laser and material science," Proc. SPIE 9666, 11th Education and Training in Optics and Photonics Conference, 966613 (5 June 2009); doi: 10.1117/12.2207951

SPIE Event: Eleventh International Topical Meeting on Education and Training in Optics and Photonics, 2009, St. Asaph, United Kingdom 


\title{
An international I nterdisciplinary Graduate School in Laser and Material science
}

\author{
Evelyne FARGIN, ICMCB, Laurent SARGER, CPMOH University BORDEAUX 1, France \\ Malte KALUZA, Stefan NOLTE, ,Freidrich Schiller University, Jena, Germany \\ Martin RICHARDSON, CREOL, University of Central Florida, FL, USA \\ Kathleen RICHARDSON, Clemson University, SC,USA,
}

\section{Introduction}

The main objective is to establish the first transatlantic Graduate School, proposing a truly international education, training and research platform in the field of Photonics and Material sciences. The wide scope of Photonics encompasses many application fields that will be mostly covered by various curricula involving Laser Optics and Material Sciences and Interactions. This cooperation will build a very efficient scientific international community able to address the 21 century challenges in Photonics and applications. Indeed, the highest level of education, namely Master and PhD , will address the so called "Skill shortage" that impact on our economy. The truly interdisciplinary theme of this graduate school is also a guarantee for the insertion of the graduate into the workforce.

The cluster culture existing in the consortium (Cluster 'Route des Lasers 'in Bordeaux, "Florida Photonic Cluster" in Orlando and "Material Science Cluster" in Clemson) will provide the necessary guidance in the market area. The workforce and international collaborations between research and Industry that will be built during this program will revive, if necessary, the economic growth of our regions through efficient research programs and technology transfer.

The success of this GRADUATE program will be assessed by the number of MS and PhD theses, eventually under cotutelle agreement, and dedicated MASTER classes are organized to fulfil this standard. The main goal of our project is then to facilitate the access of students to PhD while maintaining the excellence in curricula. This project strongly relies on the TRANSATLANTIC MASTER MILMI (Master In Laser Material Interactions) which has been settled between the partner Universities in September 2008 under the European ATLANTIS funding (2008-2012).

\section{Research based learning system}

A strong tutoring from the very beginning of the program will help the students to define an optimized path throughout the partner's institutions. The partners jointly intend to foster a work force at the highest level based on research and education. Moreover, a global culture where European and US approaches in structure and management will build a very efficient population of scientist and engineers in this high technology field

The many research teams in each institution have the required international reputation in these fields to guarantee the high level of teaching and training the students deserve. Training will be ensured by two years long laboratory internship leading to defend the "Master thesis". Then, if the research project of the student fits into the development of the themes implanted in the consortium, a PhD contract will be signed with a strong emphasis on the cotutelle framework where all the partners benefits from the activities.

Strong industrials links through the cluster's structures will also enable close collaborations within the triangle Industry/education/applied research as a keyword for innovation. This whole organization is clearly devoted to an efficient insertion of our PhD into the High Tec workforce. 


\section{History of the partnership}

Long time research history among all the partners of this consortium has focused on furthering the fundamental scientific understanding which links material science and laser material processing. These have been successful not only on the research side but also on the education side as we all early understood that we need good students able to work in very competitive area with strong employment opportunities at the highest level. All the partners associated in this proposal where the most active, since 1998, in the International REU program, funded by the National Science Foundation and national European institution for a more modest part. This program, intended to attract talented students to research activities in science, allows for long internship- in the US (UCF and later Clemson Univ.) for French (Bordeaux 1 University) and German (FSU-Jena) candidates and in the corresponding European institution for the US Undergraduate students. For the past 9 years, the educational action was focused mainly on the undergraduate level and has fostered collaboration between Chemistry Materials, Engineering and Physics departments nation-wide, both in France, Germany and in the US, This program has permitted the hiring of outstanding candidates based on their academic background, provided them a prior research experience and foreign language competency. As an outcome, we have been able at UB1, FSU, UCF and Clemson to channel appropriate candidates for graduate studies and our own PhD programs.

In order to establish a global, truly international, education and training platform in the field of Laser materials and interactions, we found easier to first address the PhD degree issue and have first proposed and obtained in 2005- a bilateral agreement for a joint doctoral supervision between UCF and UB1- a so called 'cotutelle agreement' (see annex 1). This agreement is, for the first time, a generic framework in which students can evolve in an international culture. This unique agreement is already under evaluation between Clemson University and UB1 and its form can be easily adapted to other bilateral situations. Already 7 candidates (US and French) have entered this program and we now need to build a complete, verticallyintegrated education and training effort, blending and sequentially building on educational research experiences, benefiting participants at all levels. Obviously, the master level will be very strategic in this approach as it will attract good candidates from all over the two nations .

In 2005 the two project leaders (UB1 and UCF) address this graduate level to find ways to bridge our two systems, not only vertically inside each country, but also transversely to allow the complete mobility of students. The timing was apparently quite appropriate as France recently adapted its academic program to be quite similar to the US on introducing the LMD framework. But, if each institution has a well defined set of requirements for Masters Degrees, each of which leads on naturally to their respective PhD programs, the requirements in France and respectively UCF and UC are quite different. We proposed to the FACE council a project to find common ground that will result in the development of a universal set of requirements which can allow MS students to seamlessly move between the French and US systems. Since UCF and UB1 have already established a cotutelle 'joint' agreement for PhD the establishment of common Master of Science requirements will create a truly compatible transatlantic system. Progress will be made to achieve the same agreement for individuals studying at UF and UB1 as well. This project was funded in 2005 and some keys items have been obtained that we wish to extend to the community through this proposal.

In 2008 the two project leaders (UB1 and UCF) enlarge the frame of this original proposal to bring a true European approach, in association with the prestigious of CLEMSON (SC-USA) that bring the complement in the Material science in the US and Friedrich Schiller University in Jena -Germany as a world expert in Laser technology. They introduce the concept of International Master degree and all partners commit themselves to this double degree program that was successfully presented to The EU-US -ATLANTIS program (accepted in September 2008). The EU-US ATLANTIS program of cooperation in higher Education and Training, with the funding of the EC for Europeans and the FIPSE for US students support the so called MILMI (International Master degree in Laser, Material Science and Interaction. The first mobility students( 12 students total -3 from Jena, 3 from Bordeaux, 4 from UCF and 2 from CLEMSON) will experiment this project in September 2009 by studying one year in a transatlantic University.

This approach of building a strong EU/US association in Higher education and research will fulfil the discussion and wishes explained during the ATLANTA workshop in November 2008 (Internationalization of Research and Graduate Studies and its Implications in the Transatlantic context). 
On the research side, the recent development of the partnership evolves through new structured international programs. Beginning of 2008 for example, a GIS program "LASINOF" involving all research groups in Bordeaux working in the domain of Laser-Matter interactions (2008-2013) has been funded that includes strong international mobility. We are also planning to extend to at least 8 more Master student mobility grants each year until 2012 through other funding. At least 4 mobility grants for PhD students each year are also necessary to ensure our co-tutelle programs. We globally (US and French) expect to exchange 14 Master students, $4 \mathrm{PhD}$ students, 10 staff each year until the next three years. The French ministry of higher education already supply this program with $3 \mathrm{PhD}$ supports/year for the next 4 years.

To complete the visibility of such a vertically integrated program, we found that the missing label will be a "Graduate school' that will foster and blend education with high technology in a very attractive technology, all along a structured research experience. This label of an International Graduate School will bring to the market new students with all the technical skills in the very exciting key theme of PHOTONICS and Material Science.

\section{The International Master structure}

The course consists of study-tracks jointly designed by the partner universities.

Core education in fundamentals of Physics, Optics, Laser and Material science by lectures, laboratory work and research projects, together with transferable skills (such as Social and language courses) is provided at all sites.

New trends in the second year are more specific to locations as described in the preceding table. Most of these options will be taken during the second year, along with a long internship preferably in the home institution.

A 'business' training will be offered by a team of professionals for High tech company creation. This summer school will also be available to any interested student from all over Europe and the US.

EU students: Preferably, European students will spend semester 1 in their home institution and semester 2 in one of the host institutions. Eventually, these students will stay over for the rest of the year doing the first year internship abroad and taking classes during the winter semester.

US students: For US students, choice will be open, and, as we already experienced in the UB1 - UCF agreement, master students will attend classes in year 2, eventually starting the master thesis in the European institution.

\section{Conclusion}

Building on the attractivity of the photonic research and development, we hope will strongly good students worldwide in our research areas, and especially in our lab and spinoff companies. Incentive from NSF and FIPSE for the US side as well as European institutions and Ministries of higher education are helping us to support students and researcher for their mobility but we are presently working on a more sustainable scheme to ensure long life to this photonics graduate school.

\section{Contacts:}

www.internationalopticsmasters.org

\section{France}

Pr Evelyne FARGIN- ICMCB -Universite Bordeaux 1 fargin@icmcb-bordeaux.cnrs.fr Pr Laurent SARGER- CPMOH - Universite Bordeaux 1 laurent.sarger@u-bordeaux1.fr Germany.

Pr Stefan NOLTE IAP- Friedrich Schiller University, Jena. nolte@iap.uni-jena

USA

Pr Martin RICHARDSON-CREOL-University of Central Florida Orlando mcr@creol.ucf.edu

Pr Kathleen RICHARDSON IMS Clemson University Richar3@clemson.edu 